\title{
Application of 3D Finite-Difference Time- Domain (FDTD) Method to Predict Radiation from a PCB with High Speed Pulse Propagation
}

W.J. BUCHANAN, N.K. GUPTA ${ }^{1}$ and J.M. ARNOLD ${ }^{2}$.

${ }^{1}$ Napier University, UK

2 University of Glasgow, UK

\begin{abstract}
The authors have applied the 3D FDTD technique to simulate the propagation of electrical signals within printed circuit boards (PCBs). This relates to the work currently being carried out into the propagation of very high speed digital pulses in PCBs for high bandwidth systems. Visualisation of an applied gaussian pulse from multiple sources within and outwith a PCB is shown and the techniques used to determine the system characteristics are discussed.
\end{abstract}

\section{INTRODUCTION}

The usuage of parallel processing of the 3D FDTD method allows full time-domain solutions with frequency information extracted using Fast Fourier Transform techniques.

The FDTD method uses Maxwell's equations, which define the propagation of an electromagnetic wave and the relationship between the Electric Field and the Magnetic Field. The equations are as below:

$$
\begin{gathered}
\mu \frac{\delta \mathbf{H}}{\delta t}=-\nabla \times \mathbf{E} \\
\varepsilon \frac{\delta \mathbf{E}}{\delta t}+\mathbf{J}=\nabla \times \mathbf{H} \\
\nabla \cdot \mathbf{E}=\rho \\
\nabla \cdot \mathbf{H}=0
\end{gathered}
$$

The first two equations show that a change in electric field $(\mathbf{E})$ produces a magnetic field $(\mathbf{H})$ and vice-versa. The third equation shows that the electric field $(\mathbf{E})$ is related to electric charge $(\rho)$ and the last equation shows that the magnetic field relates to magnetic charge (although it does not exist).

FDTD method has two main advantages over empirical analysis. Firstly, it provides a direct solution to Maxwell's equations without much complexities. Secondly, it takes into account all fields (electric and magnetic) in a threedimensional model.

FINITE DIFFERENCE TIME-DOMAIN (FDTD) METHOD
The FDTD method makes use of the increased power of today's computers to provide full time-domain solutions. Maxwell's continuous mathematical equations can be converted into a discrete form to allow them to be implemented on a digital computer. This results in the determination of frequency responses over a wide spectrum, Other methods would normally require different models and/or techniques for different frequency spectra.

For uniform, isotropic and homogeneous media Maxwell's curl equations can be simplified as:

$$
\begin{aligned}
\mu \frac{\delta \mathbf{H}}{\delta t} & =-\nabla \times \mathbf{E} \\
\varepsilon \frac{\delta \mathbf{E}}{\delta t} & =\nabla \times \mathbf{H}
\end{aligned}
$$

By applying appropriate boundary conditions on sources, conductors and mesh walls an approximate solution of these equations can be found over a finite three-dimensional computational domain. Taking an example of the first equation in the $i$ direction:

$$
\mu \frac{\Delta H_{x}}{\Delta t}=\frac{\Delta E_{y}}{\Delta z}-\frac{\dot{\Delta} E_{z}}{\Delta y}
$$

The central difference approximation is used on both the time and space first-order partial differentiations in order to obtain discrete approximations. This gives:

$$
\mu \frac{H_{x i, j, k}^{n+1 / 2}-H_{x i, j, k}^{n-1 / 2}}{\Delta T}=\frac{E_{y i, j, k}^{n}-E_{y i, j, k-1}^{n}}{\Delta z}-\frac{E_{z i, j, k}^{n}-E_{z i, j-1, k}^{n}}{\Delta y}
$$

Rearranging gives the following: 


$$
H_{x i, j k}^{n+1 / 2}=H_{x i, j k}^{n-1 / 2}+\frac{\Delta t}{\mu \Delta z}\left[E_{y, j, j k}^{n}-E_{y, i, j-1]}^{n}\right]-\frac{\Delta t}{\mu \Delta y}\left[E_{z i, j, k}^{n}-E_{z i-1, j, k}^{n}\right]
$$

The permittivity and the permeability are set to the approximate values depending on the location of each field component. Half time steps indicate that $\mathrm{E}$ and $\mathrm{H}$ are alternately calculated in order to achieve central differences for the time derivatives. This results in six equations similar to the one given above. These define the $\mathrm{E}$ and $\mathrm{H}$ fields, in a discrtete in the $x, y$ and $z$ directions.

The 3D FDTD process is shown in Figure 1.

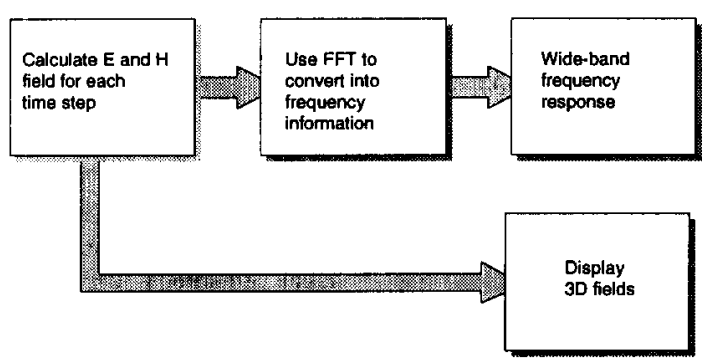

Figure 1: FDTD Method

\section{Problem conception}

The structure simulated in this paper is a PCB with four sources with copper tracks. Figure 2 shows the physical structure of the PCB. It consists of a substrate layer, such as Duroid (dielectric constant of 2.2), and a ground plane below this layer. A copper layer is formed by etching the top of the substrate to give the required pattern.

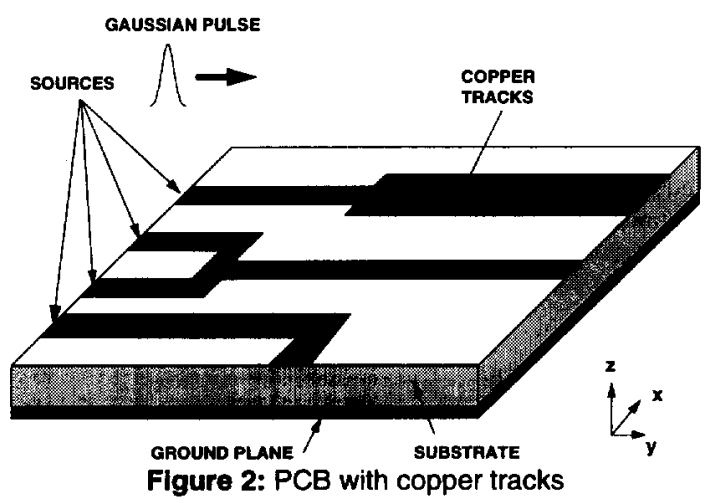

This structure can be analysed using the 3D FDTD method by taking into account the following considerations.

The relative dielectric constants in the air above the subtrate, and within the substrate are $\varepsilon_{\mathrm{r} 1}$ and $\varepsilon_{\mathrm{r} 2}$ respectively. At the interface between the air and the substrate, the relative dielectric constant approximated as the average of the two, i.e.

$$
\frac{\varepsilon_{r 1}+\varepsilon_{r 2}}{2}
$$

A Gaussian pulse is applied to the source. This is used as its frequency spectrum is also Gaussian and will provide frequency-domain information from DC to the desired cutoff frequency by adjusting the width of the pulse. This is chosen to be at least 20 points per wavelength at the highest frequency represented significantly in the pulse.

The electric field applied to the source has only a field which is perpendicular to the ground plane. Figure 3 shows a Gaussian pulse which is delayed by 30 time steps.

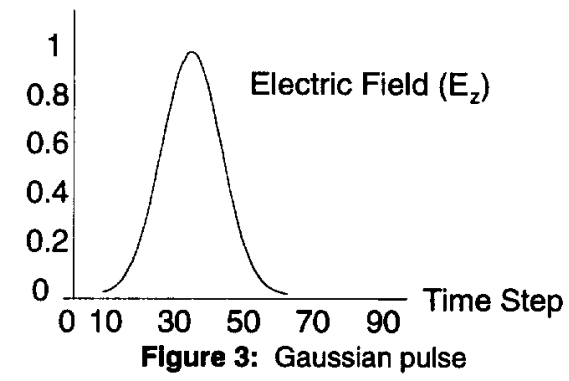

Frequency information is extracted by conducting a Fast Fourier Transform (FFT) on the transient response. For example to determine the reflection coefficient of the track a point is chosen at the source and the reflected wave is monitored. If the radiation pattern is required points are taken in free-space.

The PCB has a conducting ground plane and a single dielectric substrate with a copper layer etched for the required shape. In FDTD method these electrical conductors are assumed to be perfect and have zero thickness. The tangential electric field components that lie on the conductors are thus assumed to be zero. Figure 4 shows that the $E$ field components on the conductor will be zero in the $x$ and $y$ direction.

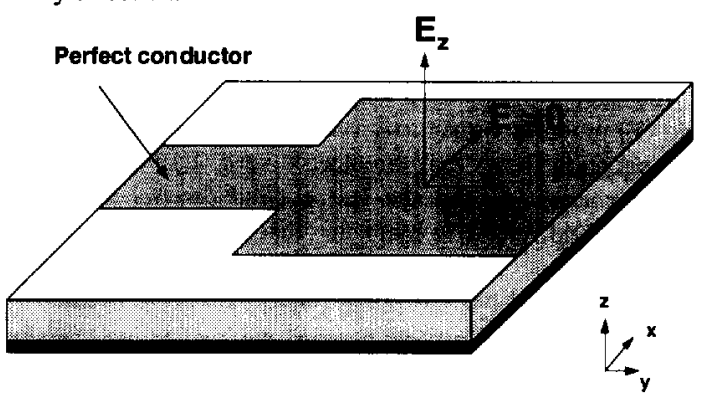

Figure 4: Conductor treatment

Six absorbing mesh boundary walls are placed around the model due to limitations in memory and speed. The ground plane and its tangential electric fields will be set to 0 and the tangential electric fields on the other five mesh walls 
must be specified so that a wave propagating against them does not reflect back. For the structure simulated in this paper the pulses will be normally incident on the mesh walls. This leads to simple approximation for continuous absorbing boundary conditions. The tangential fields on the absorbing boundaries will then obey the one-dimensional wave equation in the direction normal to the mesh wall. For the normal $y$-direction wall the one-dimensional wave equation may be written as:

$$
\left(\frac{\partial}{\partial y}-\frac{1}{v} \frac{\partial}{\partial t}\right) E_{\tan }=0
$$

This equation is Mur's first approximate absorbing boundary condition and this may be made discrete to give:

$$
E_{0}^{n+1}=E_{1}^{n+1}+\frac{v \Delta t-\Delta y}{v \Delta t+\Delta y}\left(E_{1}^{n+1}-E_{0}^{n}\right)
$$

where $E_{0}$ represents the tangential electric field on the mesh wall and $E_{1}$ the field one grid point within the mesh wall. Similar equations can be derived for the other five absorbing mesh walls. This method does not take into account fringing fields which are propagating tangential to the walls. The absorbing boundary must be placed well away from any fringing fields.

The maximum time step that may be used is limited by the stability restriction of the finite difference equations. This is shown below.

$$
\Delta t<\frac{1}{c}\left[\frac{1}{\Delta \mathrm{x}^{2}}+\frac{1}{\Delta \mathrm{y}^{2}}+\frac{1}{\Delta \mathrm{z}^{2}}\right]^{-1 / 2}
$$

$\mathrm{c}$ is the speed of light $(300,000,000 \mathrm{~m} / \mathrm{s})$ and $\Delta \mathrm{x}, \Delta \mathrm{y}$ and $\Delta \mathrm{z}$ are the dimensions of the unit cell.

A Fourier Transform is used to extract frequency information from the transient response. The scattering parameters $S_{j k}$ may be obtained by simple Fourier transform of the transient waveforms as

$$
S_{j k}(\omega)=\frac{F F\left(V_{j}(t)\right)}{F F\left(V_{k}(t)\right)}
$$

\section{RESULTS}

The simulated PCB has a width of $38.9 \mathrm{~mm}$, a length of 40 $\mathrm{mm}$, a substrate thickness of $0.8 \mathrm{~mm}$ and each time-step is approximately 0.5 picoseconds. A $100 \times 100 \times 16$ grid was used as the E-field plots in the z-direction are shown in Figures 7 16. These show the electric field plots intensity in the $\mathrm{z}$ direction and are measured just above and below the copper tracks. The results were obtained using a 4-transputer array connected to a 486-based PC.
The simulation time was reduced to almost one-quarter of that for the equivalent sequential machine. A relatively small amount of time was spent with inter-transputer communications. The model used assumes a match between the source and the copper tracks and an absorbing boundary around on the outer walls of the problem. These values will not be totally accurate as the FDTD method does not take into account conduction or dielectric losses.

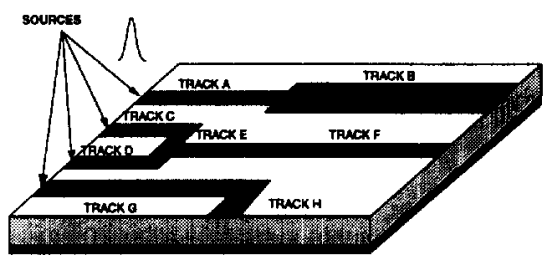

Figure 5: Conductor treatment

The following description relates to the track configuration of Figure 9. Source pulses, in Figure 7, have entered the structure and are propagating along the tracks $A$, $C, D$ and F. In Figure 8, the pulses within tracks $C$ and D encounter track E. In Figure 9, two negative pulses are reflected from this and propagate back through tracks $\mathrm{C}$ and D.

The pulse in track A enters track B and spreads outwards, this is shown in Figure 10. A negative pulse is reflected from the junction between A and $B$ (the impedance of track $B$ is less than A). Figure 11 shows that the pulse traveling in track $G$ changes direction and travels throught track $H$ Figure 12 shows a negative pulse traveling back along track $A$ and the pulse traveling in track $G$ being absorbed at the hearside wall. Figure 13 shows that after 820 time steps all the energy has been absorbed.

Figures 14-16 show the electric field just above the PCB. The z-component of the electric field directly above the tracks will be negative as the electric field lines point into the conductors, as illustrated in Figure 6.

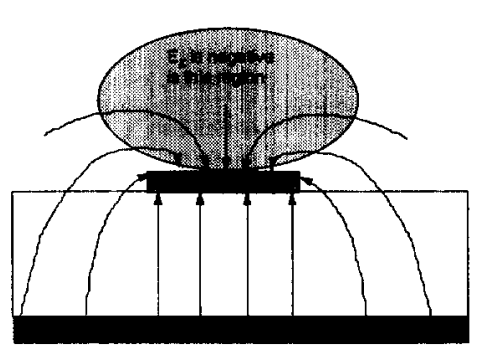

Figure 6: Electric fields around a track 


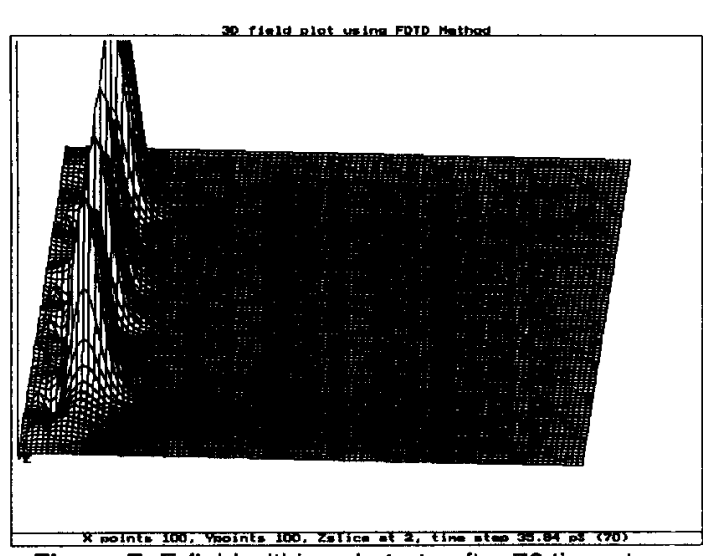

Figure 7: E-field within substrate after 70 time steps

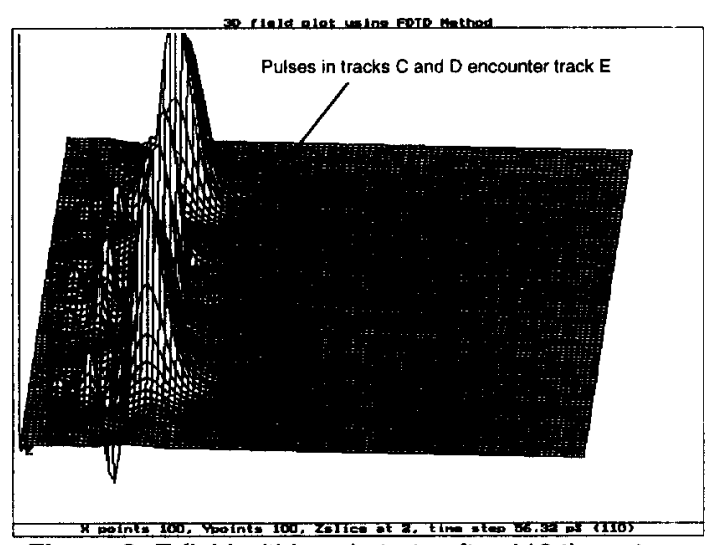

Figure 8: E-field within substrate after 110 time steps

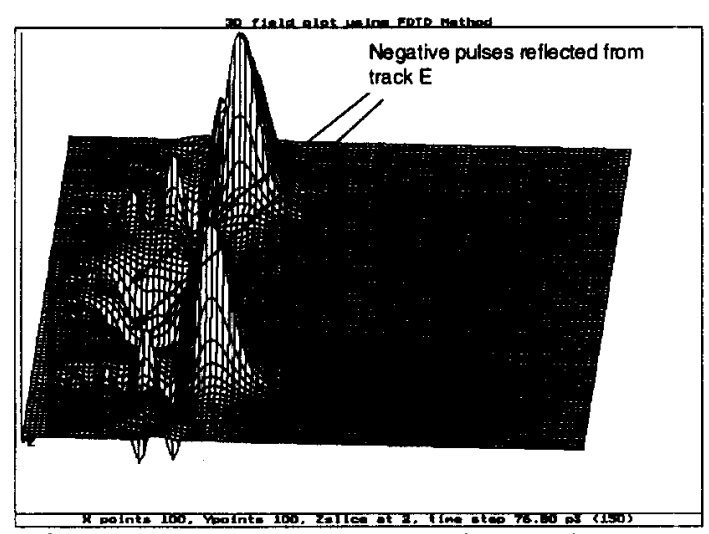

Figure 9: E-field within substrate after 150 time steps

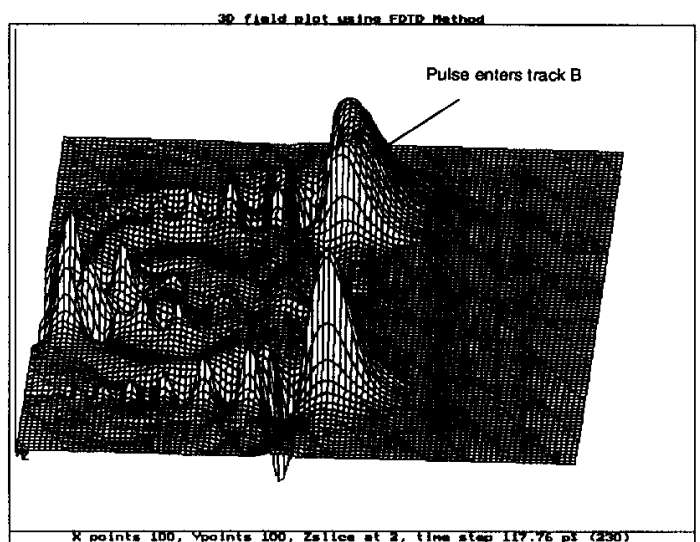

Figure 10: E-field within substrate after 230 time steps

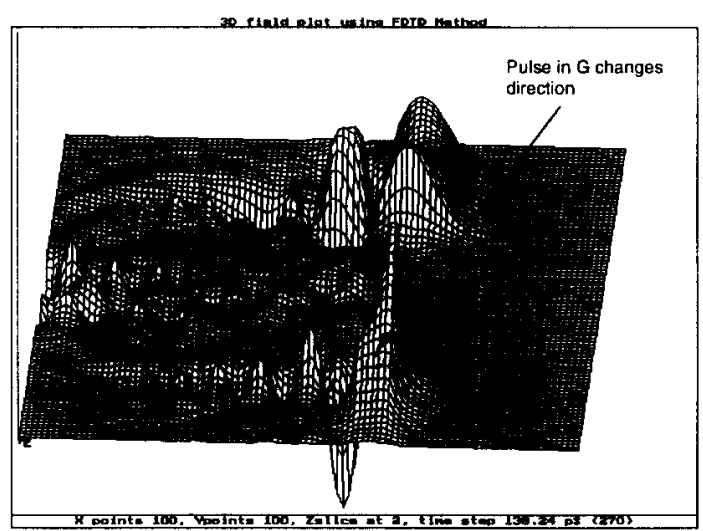

Flgure 11: E-field within substrate 270 time steps

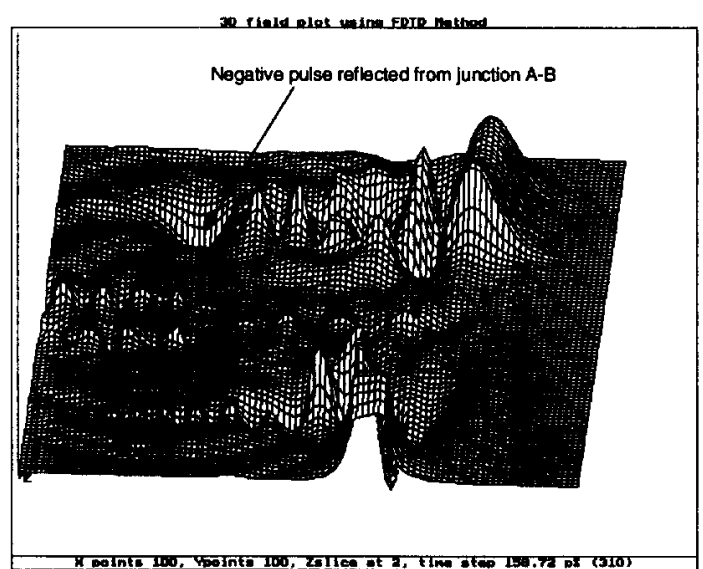

Flgure 12: E-field within substrate 310 time steps 


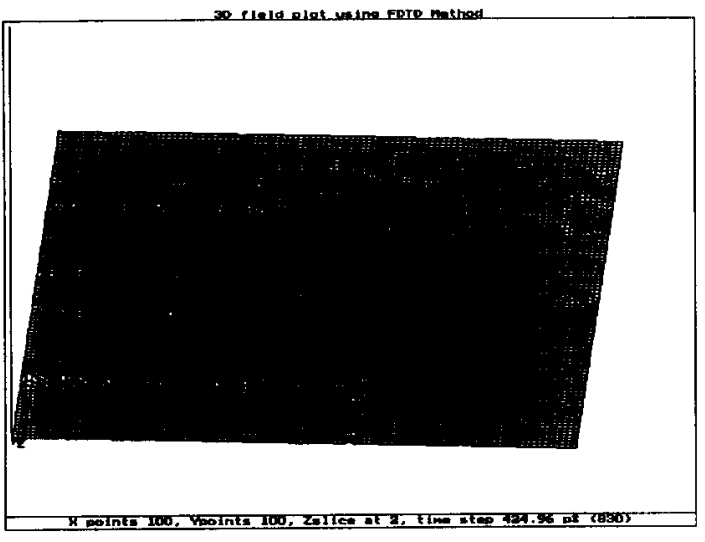

Figure 13: E-field within substrate after 820 time steps

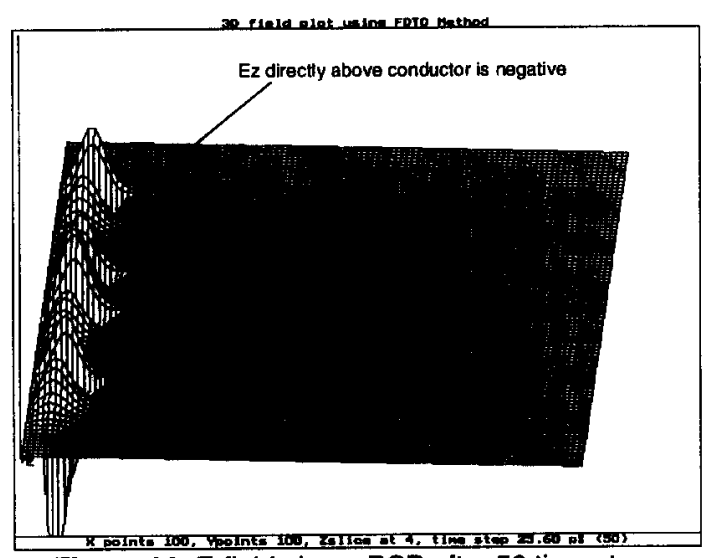

Flgure 14: E-field above PCB after 50 time steps

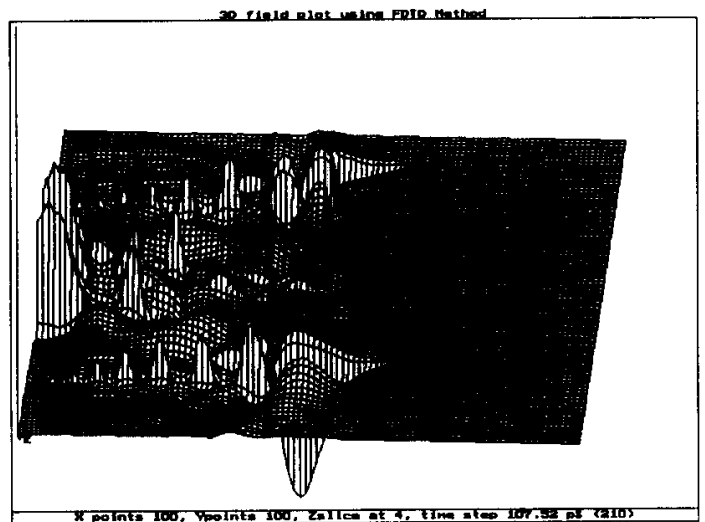

Figure 15: E-field above PCB after 210 time steps

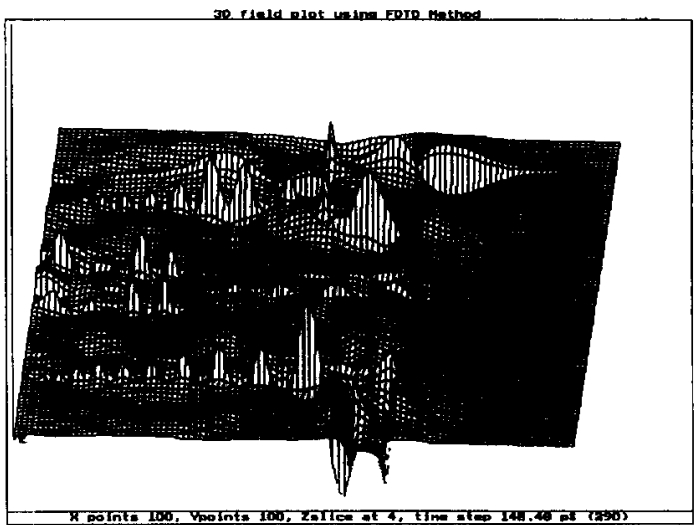

Figure 16: E-field above PCB after 230 time steps

\section{CONCLUSIONS}

The results obtained clearly show the propagation and reflection of Gaussian pulses appropriate to their position in the structure and time. These can be used to determine all required frequency characteristics from $D C$ to the required upper frequency with no change to the model for different frequency spectra.

The authors are convinced that parallel processing is an efficient and accurate technique for the simulation of complex structures by the method of FDTD to determine electrical parameters and can be used on limited memory computer systems with transputer arrays.

\section{ACKNOWLEDGEMENT}

The authors wish to thank Prof. D. Lidgate, the Head of the Department and Prof. A.E.A Almaini, Associate Head of the Department of Electrical, Electronic and Computer Engineering at Napier University, Edinburgh, UK for the use of facilities.

\section{BIBLIOGRAPHY}

D Sheen, S Ali, M Abouzahra, and J Kong,"Application of Three-Dimensional Finite-Difference Method to the Analysis of Planar Microstrip Circuits",IEEE MTT, Vol 38,pp849-857,July 1990.

$\mathrm{X}$ Zang,J Fang and $\mathrm{K}$ Mei,"Calculations of the dispersive characteristics of microstrips by the FDTD method",IEEE MTT,vol 26,pp 263-267,Feb 1988.

V SvetJana, K Yee and X Mei,"A Subgridding Method for the Time-Domain Finite-Difference Method to Solve Maxwell's Equations",IEEE MTT, Vol 39,No 3, March 1991.

Shibata T, Havashi T and Kimura T,"Analysis of microstrip circuits using three-dimensional full-wave electromagnetic field analysis in the time-domain", IEEE MTT, vol 36,pp 1064-1070, June 1988.

A Taflove, "The Finite-Difference Time-Domain Method for Electromagnetic Scattering and Interaction Problems",JEEE Trans. Electromagnetic Compatibility, vol EMC-22,pp191-202,Aug 1980.

Railton CJ, Richardson KM, McGeehan JP and Elder KF, "The Prediction of Radiation Levels from Printed Circuit Boards by means of the FDTD Method", IEE's International Conference on Computation in Electromagnetics, Savoy Place, London, November 1991.

WJ Buchanan, NK Gupta. "Parallel Processing Techniques in EMP Propagation using 3D Finite-Difference Time-Domain (FDTD) Method". Journal of Advances in Engineering Software, February 1994. 